\title{
An Automatic Antenna Tuning System using only RF-Signal Amplitudes
}

\author{
E.L. Firrao, A.J. Annema, and B. Nauta
}

\begin{abstract}
The operating environment of mobile phones fluctuates continuously, due to changing handling conditions and nearby objects. The resulting fluctuations in antenna impedance cause both a decrease in link quality and a higher standing wave ratio, that requires more robust and hence less efficient PA implementations. In this paper an automatic antenna tuner system for handheld applications is presented that uses two series reactances combined with 3 simple RF-peak detectors to sense both reactive and real impedance mismatches. The control loop only requires low-frequency electronics which makes it low cost, low power and relatively easy to integrate. Measurements on a demonstrator system show correct behaviour for VSWR up to 10.
\end{abstract}

Index Terms-Impedance matching, antenna tuning, high efficiency power amplifiers, wireless communication systems.

\section{INTRODUCTION}

Mobile devices are widely used today, with as main technology drivers --- for the RF front end --- better performance at lower power consumption and lower cost. The antennas used are typically narrow band, small, high-Q devices that are detuned by any nearby impedances formed by e.g. hands, the head, tables and more [1]-[3]. RF front ends are typically designed under the assumption of a $50 \Omega$ antenna impedance, for which maximum efficiency, talk-time, quality of the link, and maximum life-time are obtained. Due to detuning of the narrow band antenna, significant degraded efficiency and life-time can be observed [2]-[4].

This paper presents an automatic antenna tuner system that can tune fluctuations in antenna impedances, resulting in higher (overall) efficiency and lower demands on robustness aspects for front end RF blocks. Lower robustness requirements translate into lower demands on design margins that may be exchanged for higher front end efficiency. A number of requirements on antenna tuners can readily be formulated:

- Environmental fluctuation for antennas occur on a time scale of ms which is slow compared to the RF signals in the $\mathrm{GHz}$ range [5]. Consequently a relatively slow control loop is sufficient.

- The insertion loss of an antenna tuner must be small for efficiency reasons.

- The demands on the electronics in the antenna tuner system must be low to simplify implementations and to minimize the power consumption of the control loop itself.

- The antenna tuner system must be able to correct for a wide range of (heavily detuned) antenna

The authors are with the IC-Design Group, CTIT, University of Twente, Enschede, The Netherlands. E.L. Firrao is now with Thales, Hengelo, The Netherlands. This work was funded by NXP-semiconductors. impedances.

Note that by using an automatic tuner, the bulky and expensive isolators that are widely applied to absorb reflected power become redundant [4].

The paper is arranged as follows. Section II presents an analysis of the changing antenna impedance and of RF front end performance. The automatic antenna tuning system is described in section III. Measurements on demonstrator implementations are shown in section IV. The conclusions are summarized in section $\mathrm{V}$.

\section{ANTENNA IMPEDANCE AND FRONT END PERFORMANCE}

It is well known from literature that the antenna impedance is affected by any nearby impedance, such as the user's hand and/or head [1]-[3]. For this paper, many measurements of the antenna impedance were done to clearly identify the antenna impedance range and its sensitivity to the environment. Two types of widely used antennas were used: a planar inverted-F antenna (PIFA) and a ceramic antenna. A large number of typical handling positions for the two antennas were considered for measurements of impedances and sensitivities.

For both types of antenna the measurements show significant detuning of the antenna mainly due to a dramatic change of the imaginary part of the impedance. The real part of the antenna impedance appears to be much less sensitive to handling conditions.

The impedance $Z_{\text {load }}$ of the antenna roughly lies in the range $30 \Omega<\operatorname{Re}\left(Z_{\text {load }}\right)<100 \Omega$ and $0<\operatorname{Im}\left(Z_{\text {load }}\right)<100 j \Omega$. The $\mathrm{RF}$ front end performance depends heavily on the antenna impedance. Figure 1 shows a simplified block diagram for the RF front end of a single band mobile phone. A single band front end is considered here only for simplicity reasons; extending the analysis towards multi-band systems is straight forward. The area covered by the reflection coefficient of the antenna is shown in the Smith-chart at the right hand side of Figure 1 for the GSM 900 and GSM 1800 bands; the maximum VSWR for this range is about 10 .

In a typical design [7]-[8] the RF power amplifier is assumed to be loaded with a real impedance while the antenna impedance is assumed to be $50 \Omega$. Transformation of the assumed $50 \Omega$ to a lower real impedance level required by the PA is not addressed in this paper as it is merely an extra (fixed) matching stage that is irrelevant in the context of automatic antenna tuners. In the remainder of this paper we assume therefore --- without loss of generality --- that the PA includes a matching network that ideally sees $50 \Omega$ loads. Because the phase shift of each front end block is strongly design dependent, any (design-specific, fixed) extra phase 
shift $\theta$ must be taken into account. Consequently the impedance of the antenna as seen by the PA equals the antenna impedance with some fixed (design dependent) excess phase shift $2 \theta$. The area covered by the possible antenna reflection coefficients on a Smith chart at the antenna connector then translates into a full circle at the RF power amplifier output, see the leftmost Smith-chart in Figure 1.

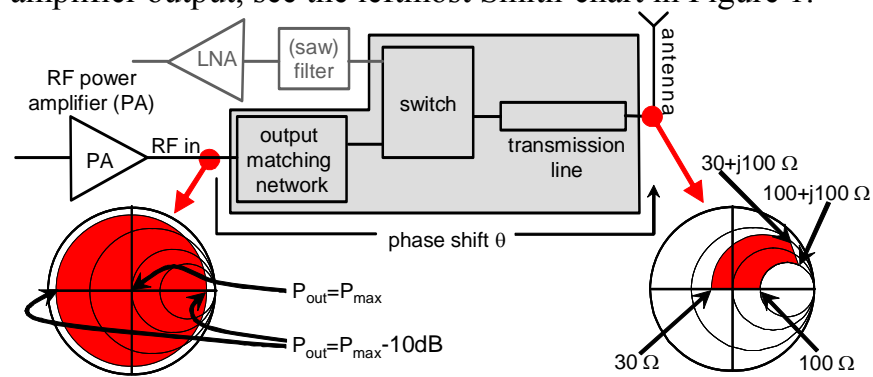

Figure 1 The RF front end: the antenna impedance seen by the PA equals the transformed antenna impedance with a design-dependent excess phase shift $\theta$.

An RF power amplifier is designed to deliver power in a certain resistive load, represented here by the centre of the Smith chart. With other load impedances, load-pull analysis [7] shows that the maximum output power is lower than the maximum by an amount $P_{\text {drop }}$ :

$$
10 \log \left(\frac{1+\left|\Gamma_{\text {antenna }}\right|^{2}}{1-\left|\Gamma_{\text {antenna }}\right|^{2}}\right) \leq P_{\text {drop }}[d B] \leq 10 \log \left(\frac{1+\left|\Gamma_{\text {antenna }}\right|}{1-\left|\Gamma_{\text {antenna }}\right|}\right)
$$

where $\Gamma_{\text {antenna }}$ is the (complex) antenna reflection coefficient. For an antenna VSWR $=10$, see Figure 1, the corresponding reflection coefficient $\left|\Gamma_{\text {antenna }}\right|=0.818$ and $P_{\text {drop }}$. is then $7 \mathrm{~dB}$ to $10 \mathrm{~dB}$. Note that the 2 boundaries in (1) correspond to a imaginary and real $\Gamma_{\text {antenna, }}$, respectively. For an antenna VSWR of 2 the power drop can be already $3 \mathrm{~dB}$ which is significant for mobile applications. In Figure 3 a $1.5 \mathrm{~dB}$ contour is included. To be able to transmit at full power levels as defined by (e.g. GSM) standards, without compensating impedance variations with automatic antenna tuners, the PA must be designed for much higher than maximum nominal power levels which significantly decreases efficiency and increases costs [7].

\section{AUTOMATIC ANTENNA TUNERS}

An automatic antenna tuner is a system able to transform the unpredictable or badly defined load impedance to a well

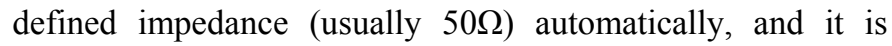
mainly made up of a tunable matching network, an impedance sensor and a control network. The tunable matching network is typically a ladder network, while the sensor provides some indication of the (tuned) antenna impedance. In this work, the main focus is on efficient impedance measurements and on an efficient control loop; the work is applicable to any tunable matching network.

A number of automatic antenna tuners can be found in literature. These can be categorized by either the way adaptation is done or by the way the impedances (or the impedance-mismatch) is measured. Roughly a number of classes can be identified:
- Adaptation methods based on analytic computation that assume that the impedance can be measured exactly [9]. Usually this requires impedance measurement at RF-frequencies and a computationally expensive control loop.

- Adaptation methods that use the sign of the phase difference to tune a reactive component to zero [4][10][11]. These methods usually employ RF-phase detectors [4][12] or Foster-Seeley discriminators [10][11] and usually are zero-crossing methods: the optimum then is a zero phase difference signal.

- Adaptation methods that minimize a pre-specified figure of merit e.g. reflected power or VSWR. These are mainly gradient descent like methods [13]-[15] or trial\&error methods [16][17].

The disadvantage of exact computation of the state of the adaptive matching network is that the antenna impedance must be measured exactly, which usually involves voltage and current measurements at RF-frequencies. This in turn puts high demands on detection circuits and hence results in relatively high power consumption for the detection circuitry.

Gradient decent methods are not pursued because of the need for the derivation of some sensitivity measure of matching network components. Adaptation based on direct information usually is much more efficient in terms of controlloop complexity. The trial\&error methods are not implemented because any knowledge based method can outperform them in antenna tuner systems.

To avoid computational expensive control mechanisms, in this work we use a zero-crossing adaptation algorithm, while for power efficiency reasons and cost reasons we use quasiDC measurable properties of RF signals (signal amplitudes) to measure the RF antenna impedance. The impedance measuring electronics and the (strongly related) control algorithm are introduced below.

\section{A. The concept}

The concept of the automatic antenna tuning system is shown in Figure 2. Tuning is done in two steps: first the imaginary part of the load impedance is tuned to (almost) zero using a series (or shunt) reactance, then the resulting real part is transformed to the target real value (i.e. $50 \Omega$ ) with a tunable "transformer". This two-step sequential tuning fits well to the low-power impedance measurement method that is introduced later in this paper.

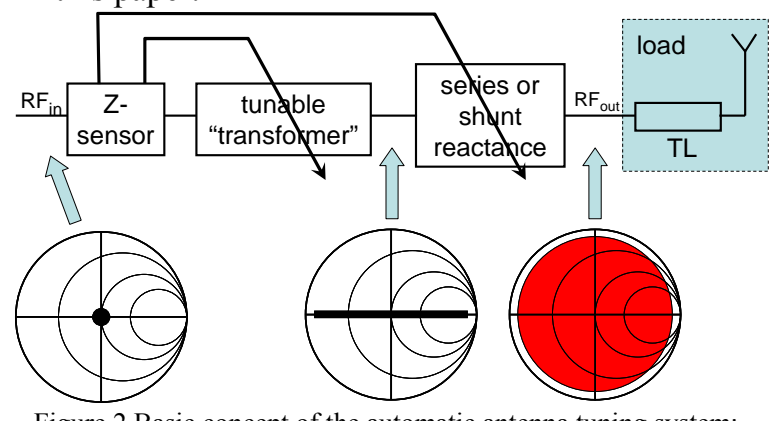

Figure 2 Basic concept of the automatic antenna tuning system: tune out the imaginary part and tune the real part separately. 
The implementation of the impedance sensor block and of the control network blocks is discussed in details in the remaining part of this section.

\section{B. The antenna impedance sensor: general setup}

Measuring the antenna impedance in some way is essential for any sensible antenna tuner system. In our system we aim at measuring the antenna impedance based on a quasi-DC property of the RF signals --- the signal amplitude --- because of power efficiency reasons and because of cost. To measure impedance, fundamentally at least three properties must be measured because voltage magnitude, current magnitude and phase must be known in some way. In our system, three signal magnitudes are measured in such a way that the antenna impedance can be derived. The schematic diagram of the total impedance sensor is shown on the left hand side in Figure 3.

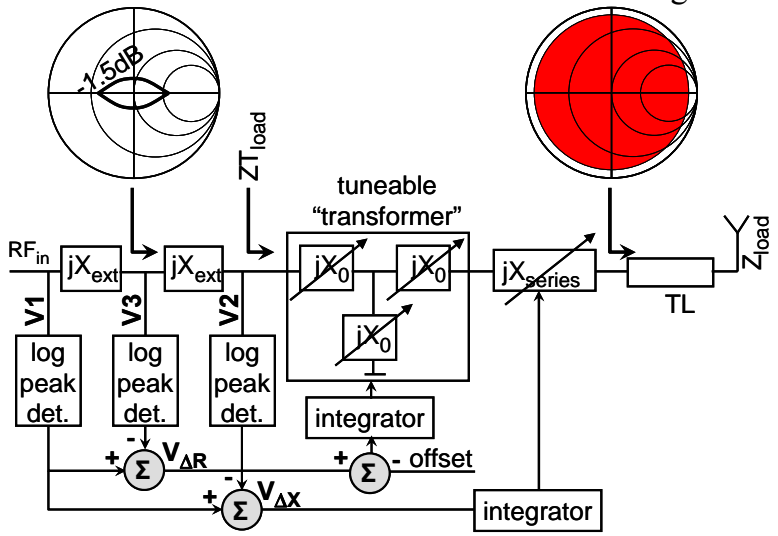

Figure 3 The automatic antenna tuning system with low frequency impedance detection; the target impedance ( $-1.5 \mathrm{~dB}$ contour) is used in section IV.A.

The impedance sensor essentially consists of two reactances and three peak detectors to detect the (low frequency) amplitude of RF signals. This detection mechanism has some similarities with the three voltmeter method [18], with as main advantage that here three quasi-DC voltages relative to ground instead of three differential voltages are measured which is easier to implement in hardware. For power level independent adaptive behavior, logarithmic peak detectors are implemented; Figure 4 shows our implementation in the experimental setup.

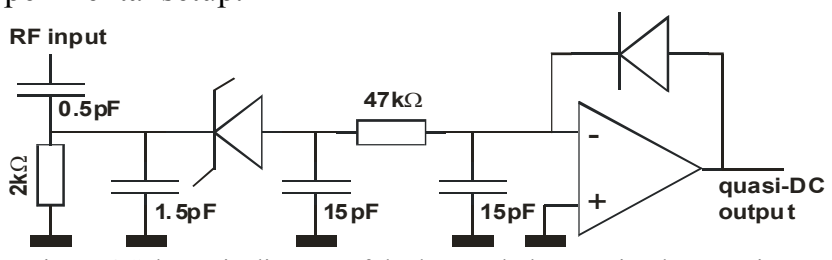

Figure 4 Schematic diagram of the log-peak detector implementation

In the peak detector circuits, only a few passives and the leftmost (Schottky)diode operate at RF frequencies. The bandwidth requirements on the remaining circuitry is determined by the bandwidth of the RF envelope and is therefore typically relatively low-frequency which enables low-power implementations and implementations in highvoltage technologies that typically are required to drive the MEMS in switchable matching networks [4].
The sensor system has two outputs: one signal $V_{\Delta X}$ indicating that the imaginary impedance (at some point in de sensor system, see below) is zero, and $V_{\Delta R}$ provides conditional information about the real part of the tuned antenna impedance.

\section{The impedance sensor: reactive part measurement}

Measuring the imaginary part of the antenna impedance is basically done by the two series reactances with values $j X_{\text {ext }}$ and the outer (logarithmic) peak detectors in Figure 3. Assuming negligible loading of the middle node, the currents in both reactances are identical. The magnitude of the voltages at nodes V1 and V2 are then proportional to the impedance at node V1 and V2. Figure 5 shows the impedance magnitudes at nodes V1 and V2 (indicated by the radius of the dotted and the solid circles, respectively) for three situations. In Figure $5 \mathrm{~b}$ the impedance magnitudes at V1 and V2 are identical; the impedance at node V3 then is real. Figure 5a shows the situation that the impedance at node V3 is capacitive for which the voltage magnitude of V2 is larger than that at node V1. Figure $5 \mathrm{c}$ shows the situation where the impedance at V3 is inductive, resulting in a larger voltage magnitude at node $\mathrm{V} 1$ than that at node V2.

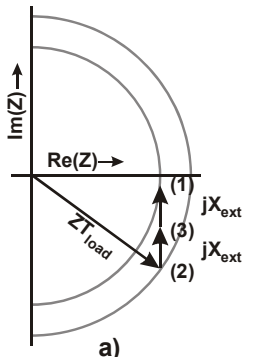

a)

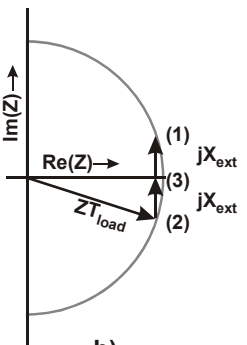

b)

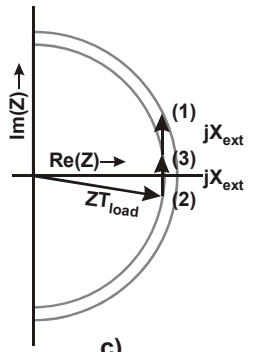

c)
Figure 5 Phasor diagrams of impedances at node V1, V2 and V3: a) capacitive reactance at $\mathrm{V} 3, \mathrm{~b}$ ) zero reactance at $\mathrm{V} 3, \mathrm{c}$ ) inductive reactance at $\mathrm{V} 3$

It follows that with ideal log-magnitude detectors:

$V_{\Delta X}=\frac{1}{2} \ln \left(1+4 \frac{\operatorname{Im}\left(Z T_{\text {load }}\right) X_{\text {ext }}+X_{\text {ext }}^{2}}{\left|Z T_{\text {load }}\right|^{2}}\right)$

This signal can be used to measure the reactive part of the (tuned) load impedance $Z T_{\text {load }}$. The signal is zero if the reactive part of $Z T_{\text {load }}$ equals $-X_{\text {ext }}$, corresponding to a zero reactance at node $V_{3}$. Note that a different $V_{\Delta X}$ value corresponds to a zero load reactance at some other (well defined) point: a zero reactance at the $R F_{\text {in }}$ node corresponds to $V_{\Delta X}=\frac{1}{2} \ln \left(1-4 X_{\text {ext }}^{2} /\left|Z T_{\text {load }}\right|^{2}\right)$.

\section{The impedance sensor: real part measurement}

Tuning of the real part of the (tuned) load impedance is done using signal $V_{\Delta R}$. Defining the impedance at node V3 as $Z_{V 3}=Z T_{\text {load }}+X_{\text {ext }}$ :

$V_{\Delta R}=\frac{1}{2} \ln \left(\frac{\operatorname{Re}\left(Z_{V 3}\right)^{2}+\left(\operatorname{Im}\left(Z_{V 3}\right)+X_{e x t}\right)^{2}}{\left|Z_{V 3}\right|^{2}}\right)$ 
In the special case that the impedance at node V3 is real, this relation simplifies to a relation that directly provides information about the (tuned) antenna:

$V_{\Delta R}\left(R_{V 3}\right)=\frac{1}{2} \ln \left(1+\left(\frac{X_{e x t}}{R_{V 3}}\right)^{2}\right)$ when $V_{\Delta X}=0$

In antenna tuning systems $V_{\Delta R}$ must be tuned to a target value $V_{\text {target } 2}=V_{\Delta R}(50 \Omega)$ to get a load equal to $R_{\text {TARGET }}=50 \Omega$. In Figure 3 this is realized by adding offset to $V_{\Delta R}$.

\section{E. The tunable matching network}

The implementation of the tunable matching network system is given in Figure 3; that of the "log peak detectors" is shown in Figure 4. The tunable "transformer" is implemented using a T-network made up by two inductors (with value $X_{0}$ ) and one capacitor (of value $-X_{0}$ ). This type of network can be seen as a lumped model of a transmission line; for exact tuning:

$$
X_{\text {series }}=-\operatorname{Im}\left[Z_{\text {load }}\right] \text { and } X_{0}=\sqrt{R_{\text {in }} \operatorname{Re}\left[Z_{\text {load }}\right]}
$$

where $Z_{\text {load }}$ is the load impedance and $R_{\text {in }}$ is the real impedance required by the $\mathrm{PA}$.

\section{F. The control network}

The signals $V_{\Delta X}$ and $V_{\Delta R}$ give a measure for the imaginary part and of (conditionally) the real part of the (tuned) antenna, respectively. We used these signals in a very simple integrating control loop, see Figure 3. Due to the relatively slowly changing environment of handheld devices (related to the signal frequencies) the performance of this simple control loop is sufficient to keep the antenna properly tuned during typical real-life handling conditions.

\section{EXPERIMENTAL RESULTS}

Two demonstrators were developed for matching any load impedance with $V S W R \leq 10$, at $900 \mathrm{MHz}$. One has sequential and discretized tuning. The discretization implies that only a limited set of (switchable) matching network states were implemented. With the sequential tuning, first the reactive part of the load is tuned and then the real part is tuned to $50 \Omega$. In this demonstrator, tuning of the real part is enabled if the reactive part is within a few times the minimum reactive tuning step size. With sequential tuning both the reactive and real tuning is monotonous towards the target impedance.

The second demonstrator implements simultaneous and continuous adaptation of the reactive and real part. Simultaneous adaptation simplifies the implementation as no threshold is required for tuning of the real part. Simultaneous adaptation can be shown to have guaranteed convergence for bounded matching network tuning ranges; the adaptation of $\operatorname{Re}\left(Z_{V 3}\right)$ however can show non-monotonous behavior. The continuous adaptation corresponds to a matching network that either can switch between many states or that implements true continuous matching "states".

For both examples, the adaptable matching network and the antenna impedance were emulated by a Maury tuner controlled by a pc. For both designs the real and imaginary part of $Z_{\text {antenna }}$ are in the ranges $R_{\text {load }} \in[5 \Omega, 500 \Omega]$ and $X_{\text {load }} \in[-250 \Omega, 250 \Omega]$, resulting in (ideally required) tuning ranges $X_{\text {series }} \in[-250 \Omega, 250 \Omega]$ and $X_{0} \in[15.8 \Omega, 158 \Omega]$. The discrete (SMD) measurement reactance $X_{\text {ext }}=19 \Omega$.

\section{A. Discretized and sequential tuning}

For the discretized and sequential tuning demonstrator, the target impedance was defined as an impedance region for which the matching was acceptable: a $-1.5 \mathrm{~dB}$ loss contour [7] was used, see Figure 3. The size of this target area, together with the initial antenna impedance region, determines the minimum number of switchable matching network states.

For the discrete implementation in order to fulfill the requirement of $-1.5 \mathrm{~dB}$ power contour, it can be derived that 120 matching network states are sufficient; we implemented 500 possible states for the switchable matching network. In the measurement setup a Maury tuner was used to emulate the total impedance of the tunable matching network and antenna. The Maury is driven by a pe that implements the control algorithm, matching network state quantization and actual control of the Maury. The detector circuitry in Figure 4 was realized on PCB using SMD components.

Many measurements were performed for both version of the control loop at various power levels (from $4 \mathrm{dBm}$ to $24 \mathrm{dBm}$ ). All measurements started with an untuned system, $X_{\text {series }}=0 \Omega$ and $X_{0}=50 \Omega$. Figure 6 shows the impedance trajectories as seen by the PA (including sensor circuitry, tunable transformer, series reactance, cables, fixtures, ...) for four extreme antenna impedances. The end of each adaptation is indicated by the arrows; the eventual "state" hops across the 3 states surrounding this end point due to the discretization.

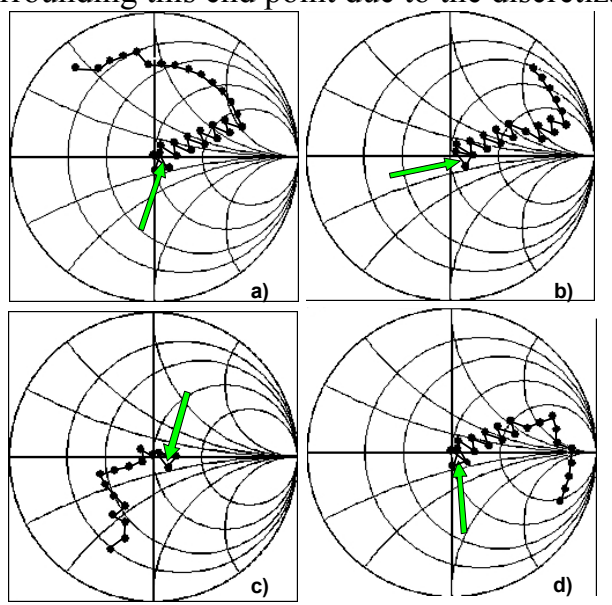

Figure 6 Measurement results of the PA-load impedance with discretized sequential tuning a) $Z_{\text {antenna }}=10-j 60 \Omega$ b) $Z_{\text {antenna }}=15-j 18 \Omega$ c)

$Z_{\text {antenna }}=100+j 100 \Omega$ d) $Z_{\text {antenna }}=5+j 18 \Omega$; arrows indicate end region center

Note that due to the sequential adaptation of the matching network, the impedance curves tend to sharply bend once the (horizontal) real axis is approached. This corresponds to setting in of the real part's tuning as soon as a sufficiently small imaginary residue is reached. 


\section{B. Continuous and simultaneous tuning}

Whereas for discretized tuning a target region must be defined (to estimate the required number of matching network states) for continuous tuning the target is set to $50 \Omega$ : the centre of the Smith chart. For simultaneous adaptation, the imaginary part monotonously goes to zero, while the convergence of the real part to $50 \Omega$ may show some over- or undershoot.

Figure 7 shows the impedance trajectories as seen by the PA for the same initial settings and antenna impedances as used for Figure 6. The end-point impedance (indicated by the arrow) is close to $50 \Omega$.

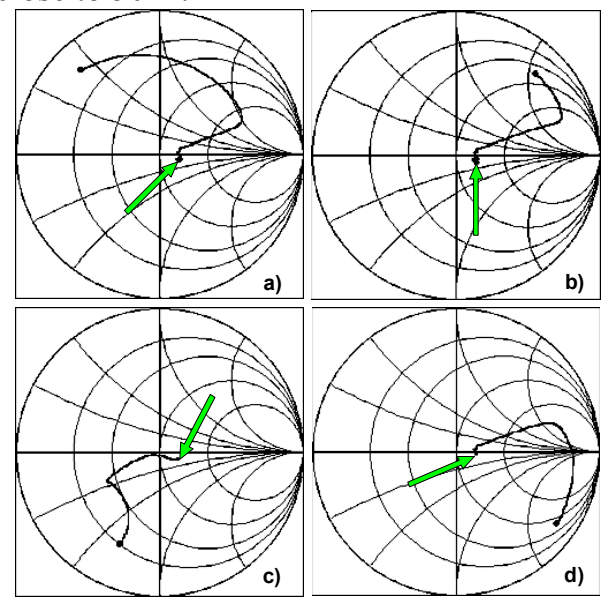

Figure 7 Measurement results of the PA-load impedance for continuous simultaneous adaptation a) $Z_{\text {antenna }}=10-j 60 \Omega$ b) $Z_{\text {antenna }}=15-j 18 \Omega$ c)

$Z_{\text {antenna }}=100+j 100 \Omega$ d) $Z_{\text {antenna }}=5+j 18 \Omega$; arrows indicate end point

\section{Discussion}

The sensor circuitry and the adaptive matching network sofar were regarded as separate blocks. Noting that both the adaptive matching network and the impedance sensor block consists of reactances, it is straightforward to (partially) merge these.

The accuracy of the discretized tuning system is typically limited by the limited number of matching network states. In our continuous tuning system the accuracy is limited by mainly:

- the residual reactive part $X_{\text {ext }}$ seen by the PA. In the proposed system we tune the impedance at node V3 to zero; by adding an offset to $V_{\Delta X}$ the zero-reactive impedance can however be shifted to the PA output.

- loading effects of the log peak detector circuitry; in our discrete implementation, the load of the peak detectors is about $0.5 \mathrm{pF}$.

Due to the low-frequency detection principle, matching requirements for the detector electronics typically are relatively easy to meet.

\section{CONCLUSIONS}

A novel automatic antenna tuning system for handheld applications is presented. This tuning system uses only magnitude information of the RF signal (at three positions) to measure the antenna impedance, while the electronics and control loop can operate at relatively low frequencies. These low frequency properties result in low power consumption and low cost implementation possibilities.

Measurements on a demonstrator implementation at $900 \mathrm{MHz}$ and at various power levels verify correct operation, and show proper convergence of the impedance as seen by the PA to values near the optimum value, starting on any impedance that result in VSWR up to 10. The end-point inaccuracy is ultimately due to the residual series reactance (compensatable) and the load impedance of the impedance sensor circuitry. Driving switchable matching networks, the inaccuracy is typically in the quantization error due to the limited number of matching states.

\section{ACKNOWLEDGMENT}

The authors thank Henk de Vries for technical assistance.

\section{REFERENCES}

[1] R.A. Sadeghzadeh and N.J. McEwan, "Prediction of Head Proximity Effect on Antenna Impedance Using Spherical Waves Expansions", Electronics Letters, Vol. 30, pp. 844-847, August 1994

[2] G.F. Pedersen, J.O. Nielsen, K. Olesen and I. Z. Kovacs, "Measured Variation in Performance of Handheld Antennas for a Large Number of Test Persons", IEEE $48^{\text {th }}$ Vehicular Technology Conference, May 1998

[3] K. Boyle, "The performance of GSM 900 antenna in the presence of people and phantoms", IEEE International Conference on Antennas and Propagation, vol. 1, pp. 35-38, March 2003

[4] A. van Bezooijen, M. de Jongh, C. Chanlo, L. Ruijs, H.J. ten Dolle, P. Lok, F. van Straaten, J. Sneep, R. Mahmoudi and A.H.M. van Roermund, "RF-MEMS based adaptive antenna matching module", in Proc. IEEE RF-IC Symposium, 2007, pp. 573-576

[5] P. Sjoblom and H. Sjoland, "An Adaptive impedance tuning CMOS circuit for ISM 2.4 GHz Band", IEEE Tr. Circuits and Systems I, vol. 52, June 2005, pp. 1115-1124

[6] Constantine A. Balanis, Antenna theory: analysis and design, John Wiley \& Sons, 1982

[7] S.C. Cripps, Advanced techniques in RF power amplifier design, Artech House, 2002

[8] Mihai Albulet, RF Power Amplifiers, Noble Publishing, 2001

[9] R.E. Collin, Foundations for Microwave Engineering, McGrill Hill

[10] P.R. Johannessen, "Automatic tuning of high Q antenna for VLF FSK transmission", IEEE Tr. Communications Systems, pp. 110-115, March 1964

[11] A.A. Castro, "Automatic Tuning System for High Power Amplifiers", IEEE Tr. Communication Technology, pp. 824-834, December 1966

[12] F.Meng, A.van Bezooijen, and R.Mahmoudi, "A mismatch detector for adaptive impedance matching", in Proc. of the 36th European Microwave Conference", pp. 1457-1460, 2006.

[13] M.M. Doss, "A fast adaptive technique for impedance matching networks", $8^{\text {th }}$ Mediterranean Electro Technical Conference, 1996, pp. 599-602

[14] J.de Mingo, A.Valdovinos, A.Crespo, D.Navarro, P.Garcia, "An RF Electronically Controlled Impedance Tuning Network Design and Its Application to an Antenna Input Impedance Automatic Matching System", IEEE Tr. Microwave Theory and Techniques, pp. 489-497, February 2004

[15] M.Thompson, J.K. Fidler, "Fast antenna tuning using transputer based simulated annealing", Electronics Letters, Vol. 36, pp. 603-604, March 2000

[16] Y.Sun, W.K.Lau, "Evolutionary tuning method for automatic impedance matching in communication systems", IEEE International Conference on Electronics, Circuits and Systems, September1998, pp. 73-77

[17] M.Thompson, J.K.Fidler, "Application of the genetic algorithm and simulated annealing to LC filter tuning", IEE Proceedings on Circuits, Devices and Systems, pp. 177-182, August 2001

[18] A.N. Plakhotnyuk, "Phase Measurement at Ultrasonic Frequencies by the Three-Voltmeter Method", Measurement Techniques, pp. 328-331, March 1968 